\title{
Level Set Segmentation of Lumbar Vertebrae using Appearance Models
}

\author{
Karl Fritscher ${ }^{1}$, Stefan Leber $^{1}$, Werner Schmölz ${ }^{2}$, Rainer Schubert ${ }^{1}$ \\ ${ }^{1}$ Institute for Biomedical Image Analysis, \\ University for Health Sciences, Medical Informatics and Technology, Hall in Tirol \\ ${ }^{2}$ University Clinic of Diagnostical Radiology, Medical University of Innsbruck \\ karl.fritscher@umit.at
}

\begin{abstract}
For the planning of surgical interventions of the spine exact knowledge about 3D shape and the local bone quality of vertebrae are of great importance in order to estimate the anchorage strength of screws or implants. As a prerequisite for quantitative analysis a method for objective and therefore automated segmentation of vertebrae is needed. In this paper a framework for the automatic segmentation of vertebrae using 3D appearance models in a level set framework is presented. In this framework model information as well as gradient information and probabilities of pixel intensities at object edges in the unseen image are used. The method is tested on 29 lumbar vertebrae leading to accurate results, which can be useful for surgical planning and further analysis of the local bone quality.
\end{abstract}

\section{Introduction}

For surgical interventions at the spine after vertebral instability exact preoperative surgical planning is crucial. It is highly important for the surgeon to gain insight into the exact 3D shape and the local quality of vertebrae in order to estimate the anchorage strength of screws or implants that are planned to be inserted into the vertebra. For this purpose the calculations of features which are able to asses the local bone quality in predefined regions of interest (ROIs) is necessary. Ideally these ROIs should be placed automatically in order to guarantee reproducible and objective results concerning the placement, size and - possibly - shape of the ROIs. For this purpose an - ideally - automatic segmentation of the vertebra is needed. In the last decade geometric deformable models based on level sets [1] became a widely used methods in medical segmentation pipelines. In [2] Leventon combined geometric deformable models with prior shape information in the level set evolution process. By introducing geodesic active regions [3] Paragios et al. also used texture information for the level set segmentation process. In [4] an approach based on level set segmentation, which is especially focusing on the segmentation of syndesmophytes in the vertebral body is introduced. In [5] a method based on the usage of shape models was proposed for the segmentation of a vertebra. In this paper we propose an approach which uses 
a level set framework in combination with appearance models for the segmentation of lumbar vertebrae. By combining level sets and appearance models the robustness of model based segmentation shall be combined with the flexibility of level set segmentation. Based on the work presented in [6] a cost function using a non-parameteric kernel based method to assess the intensity distribution at object edges will be proposed. It is the purpose of this paper to investigate if the proposed methods are feasible to provide a segmentation which can be used for further tasks concerning the planning of surgical interventions at the lumbar spine.

\section{Methods}

For the creation of the model pre-segmented images of the organ of interest are needed in order build 3D appearance models as described in [6]. Moreover, the level-set segmentation framework presented in [6] was used as a basis to combine model and image gradient based segmentation. In this work, the same speed function as in [6] was used. However, the grey scale statistics term for the MAP estimation presented in [6] was replaced by a term based on a nonparametric kernel-based method as presented in [7]. This term uses a Gaussian kernel which enables the calculation of the probability of a pixel's intensity i of belonging to a given region. This probability can be calculated as follows

$$
p\left(i \mid \phi_{M}\right)=\frac{1}{V(R)} \iint_{R} \frac{1}{\sqrt{2 \pi \sigma}} e^{\frac{-(i-I(y))^{2}}{2 \sigma^{2}}}
$$

where $\mathrm{V}(\mathrm{R})$ is the volume of the region $\mathrm{R}$ and $\sigma$ specifies the width of the Gaussian kernel. I(y) represents the intensity of a pixel at index y. $\phi_{M}$ stands for the current zero level set. In contrast to [7] where this kernel-based method is used to calculate the probability of a pixels intensity to be consistent with the whole interior of a deformable contour, in our implementation the probabilities for a given pixel intensity are calculated within 2 regions $P_{\text {in }}$ and $P_{\text {out }}$. These regions are represented by a narrow band [8] at the inside and outside of the propagating level set front. If the probability of a pixel with intensity $i$ lying on the evolving front is higher for the narrow band inside the evolving region than the probability of belonging to the outside region, the front expands outwards. If the probabilities are vice versa the front moves inwards. The speed of the evolution is proportional to the calculated difference of the two probabilities $P_{\text {in }}$ and $P_{\text {out }}$. The curve stops if the probability for a pixel with intensity $i$ for belonging to $P_{\text {in }}$ is equally high as the probability for belonging to $P_{\text {out }}$. In practice the pixels in the regions are converted to 8 bit (256 grey values). Moreover, this intensity statistics term (IST) is calculated independently in different sub-regions of the evolving front since it has to be assumed that the pixel intensities at the edge of the object of interest are not homogeneous throughout the whole surface. Furthermore the width of the narrow band in which the probabilities are calculated can be parameterized as well as the amount of the influence of the intensity statistics term on the propagation of the level set front using a weighting factor. 
Table 1. Segmentation results for datasets included in model

\begin{tabular}{lcccccc}
\hline & Known vertebrae using IST & \multicolumn{3}{c}{ Known vertebrae without IST } \\
\hline Dataset & SI & HD $(\mathrm{mm})$ & MD $(\mathrm{mm})$ & SI & HD $(\mathrm{mm})$ & MD $(\mathrm{mm})$ \\
\hline Average: & 0.932 & 6.722 & 0.512 & 0.922 & 6.746 & 0.586 \\
Sigma: & 0.013 & 2.970 & 0.130 & 0.017 & 2.553 & 0.113 \\
\hline
\end{tabular}

\section{Results}

The proposed algorithm was tested using 29 CT datasets of specimen of the spine and its surrounding muscles having an image resolution of $0.7 \mathrm{~mm}^{3}$. 19 of these datasets have been used to create statistical appearance model of the third lumbar vertebra (L3) as described in [6]. Figure 1 shows the first two modes of variation for the shape of L3 within 2 standard deviations. The first 10 modes which are representing 92 percent of the appearance variations of L3 were used for the segmentation.

The 19 datasets used to build the model and the 10 unseen datasets have been segmented twice: One time using the new intensity statistics term and one time without this term. As gold standard a manual segmentation of the vertebra performed by a medical expert was used. The initialization for the automatic segmentation was performed by manually defining a rectangular region around the vertebra of interest. Tables 1 and 2 are showing the mean value and standard deviation of three common metric values - similarity index (SI), contour mean distance (MD) and Hausdorff distance (HD) - for the comparison of automatically segmented vertebra and the gold standard. It can be observed that the usage of the intensity statistics term improved the results of the segmentation.

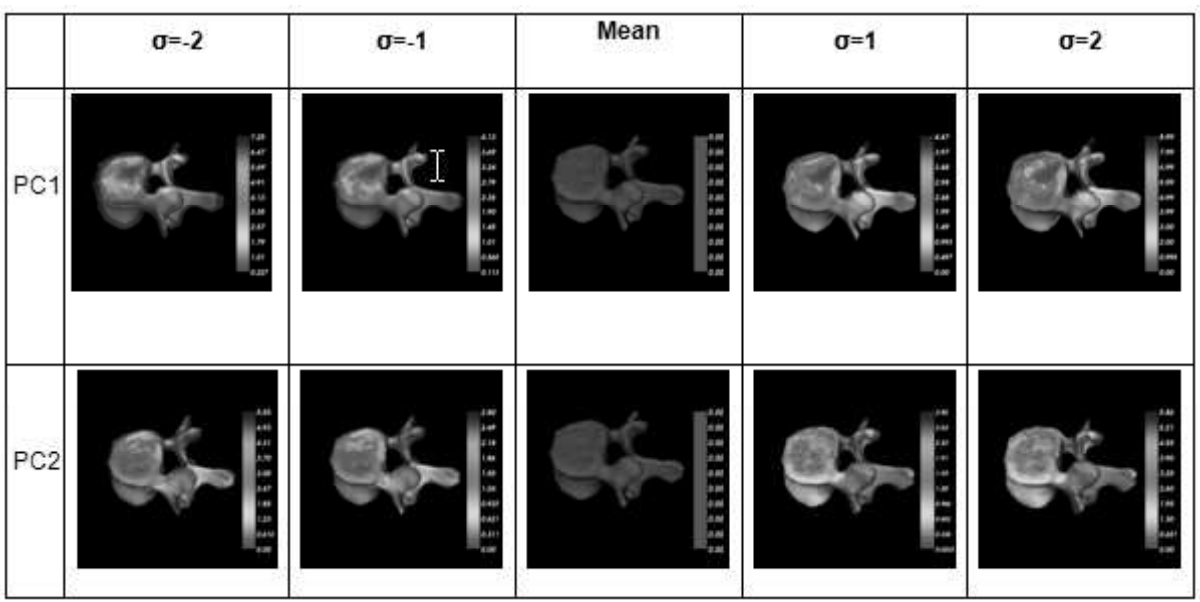

Fig. 1. First 2 modes of variation for the shape of L3 within 2 standard deviations from the mean (axial cut) 
Table 2. Segmentation results for unseen datasets

\begin{tabular}{lcccccc}
\hline & Unseen vertebrae using IST & \multicolumn{3}{c}{ Unseen vertebrae without IST } \\
\hline Dataset & SI & HD $(\mathrm{mm})$ & MD $(\mathrm{mm})$ & SI & HD $(\mathrm{mm})$ & MD $(\mathrm{mm})$ \\
\hline Average: & 0.918 & 9.249 & 0.625 & 0.903 & 9.405 & 0.8 \\
Sigma: & 0.025 & 2.638 & 0.149 & 0.032 & 2.603 & 0.282 \\
\hline
\end{tabular}

Figure 2 is showing two segmentation results. The left image is showing a vertebra that has been included in the model, the right image shows the segmentation result for a new vertebra.

\section{Discussion}

In this paper a way to combine the framework presented in [6] with a non parametric kernel based intensity statistics term was introduced. The segmentation for both the included as well as for the unseen datasets showed satisfying results with an average mean distance of $0.59 \mathrm{~mm}$ between automatic and manual segmentation performed by an expert. This is below the pixel spacing of $0.7 \mathrm{~mm}$ and the true $3 \mathrm{D}$ shape of the vertebra can be reconstructed with high accuracy suitable for surgical planning. It could be shown that the results of the segmentation could be improved by using the intensity statistics term. The segmentation faults were mainly situated in the transverse process. Since this is typically not a region, where the local bone quality must be known for surgical interventions, the segmentation is also highly suitable for the placement of adaptive ROIs using a method like e.g. presented in [9]. Since the appearance model is also reproducing the intensity distribution of the vertebra in high detail, the possibility of
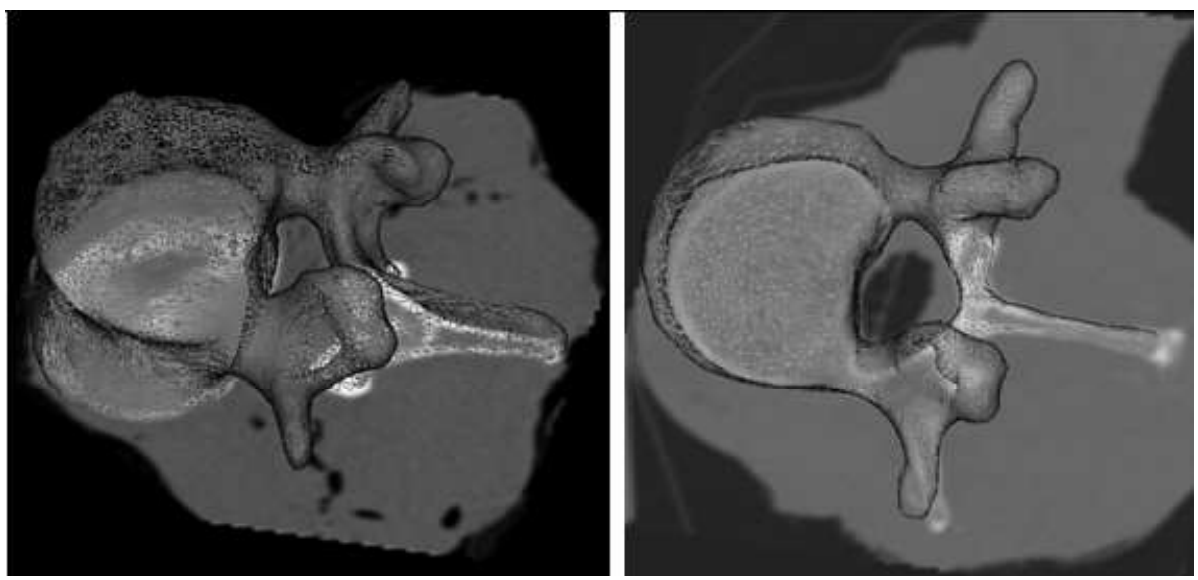

Fig. 2. Result of automatic segmentation for vertebra included in the model (left) and for unseen vertebra 
using the final estimate of the appearance parameters at the end of the segmentation process directly for further analysis of the local bone quality should be considered.

\section{References}

1. Osher S, Sethian J, James A. Fronts propagating with curvature-dependent speed: Algorithms based on Hamilton-Jacobi formulations. J Comput Phys. 1988;79:12-49.

2. Leventon ME, Grimson WEL, Faugeras O. Statistical shape influence in geodesic active contours. IEEE Comp Vis Pattern Recognit. 2000;01:1316.

3. Paragios N, Deriche R. Geodesic active regions: A new framework to deal with frame partition problems in computer vision. J Vis Commun Image Represent. 2002;13:249-68.

4. Tan S, Jinhua Y, Ward M, et al. Level set based vertebra segemntation for the evaluation of ankylosing spondylitis. Proc SPIE. 2006;6144:07/01-10.

5. Fritscher KD, Schubert R. 3D image segmentation by using statistical deformation models and level sets. J Comp Assist Radiol Surg. 2006;1:123-35.

6. Fritscher KD, Gruenerbl A, Schubert R. 3D image segmentation using combined shape-intensity prior models. J Comp Assist Radiol Surg. 2007;1:341-50.

7. Huang X, Metaxas D, Chen T. Metamorphs: Deformable shape and texture models. IEEE Comp Vis Pattern Recognit. 2004;1:496-503.

8. Chopp DL. Computing minimal surfaces via level set curvature flow. J Comput Phys. 1993;106(1):77-91.

9. Fritscher KD, Schuler B, Grünerbl A, et al. Assessment of femoral bone quality using co-occurrence matrices and adaptive regions of interest. Proc SPIE. 2007;6514:1K19 . 